\title{
Review
}

Michiyo Yanase*, Takashi Kuriki

\section{Synthesis and functionalization of glucan dendrimer, and its application as a drug delivery system carrier}

https://doi.org/10.1515/amylase-2021-0005

Received July 1, 2021; accepted September 22, 2021.

\begin{abstract}
Glycogen is a natural polysaccharide with a dendrimer structure, in which glucose is frequently branched and polymerized. Functionalizing the numerous non-reducing ends on the molecular surface of glycogen could be expected to enable its use in various fields. We developed a method for enzymatically synthesizing a suitable form of glycogen from sucrose by using sucrose phosphorylase and branching enzyme, both of which belong to the $\alpha$-amylase family, as well as glucan phosphorylase. We refer to this enzymatically synthesized glycogen as the glucan dendrimer (GD). We then selectively modified the non-reducing ends on the surface of GD particles by using the reaction of glucan phosphorylase with various hexose 1-phosphates. Modifying the nonreducing ends of GD with glucuronic acid or glucosamine added negative and positive charges to the GD particles. In addition, we found that glucuronic acid and/or glucosamine residues at the non-reducing ends can be used to covalently conjugate functional substances, such as sugar chains, proteins, and peptides to the surface of GD particles. GD and modification of its non-reducing ends represent versatile platforms for pharmaceutical applications of polysaccharides.
\end{abstract}

Keywords: glycogen; dendrimer; glucan phosphorylase; sucrose phosphorylase; branching enzyme.

\section{Abbreviations}

AqGP, glucan phosphorylase from Aquifex aeolicus; DDS, drug delivery system; GA, glucoamylase; Gal,

\footnotetext{
*Corresponding author: Michiyo Yanase, Mechanism-based Research Laboratory, Ezaki Glico Co., Ltd., 4-6-5 Utajima, Nishiyodogawa, Osaka 555-8502, Japan, E-mail: michiyo.yanase@glico.com Takashi Kuriki, Research Fellow, Ezaki Glico Co., Ltd., 4-6-5 Utajima, Nishiyodogawa, Osaka 555-8502, Japan
}

galactose; Gal1P, galactose 1-phosphate; GalN1P, galactosamine 1-phosphate; GD, glucan dendrimer; Glc, glucose; Glc1P, glucose 1-phosphate; $\mathrm{Glc}_{2}$, maltose; $\mathrm{Glc}_{3}$, maltotriose; $\mathrm{Glc}_{4}$, maltotetraose; GlcA1P, glucuronic acid 1-phosphate; GlcN1P, glucosamine 1-phosphate; GlcNAc, $N$-acetylglucosamine; GlcNAc1P, $N$-acetylglucosamine 1-phosphate; HPAEC-PAD, high-performance anionexchange chromatography with pulsed amperometric detection; Man1P, mannose 1-phosphate; PotGP, glucan phosphorylase from potato.

\section{Introduction}

Glycogen is a highly branched glucose polysaccharide that serves as a form of energy storage in animals and bacteria. The molecular surface of glycogen has numerous nonreducing ends. We hypothesized that functionalizing these ends could enable various applications of glycogen in the production of not only conventional food but also cosmetics and pharmaceuticals, among other fields. In this paper, we introduce our research on the enzymatic synthesis of glycogen-like polysaccharides with tightly controlled molecular weight and particle size and on the technique for selectively modifying their non-reducing ends.

\section{Synthesis of glycogen- like polysaccharides with phosphorylase}

\subsection{Development of carbohydrate synthesis and production technology with phosphorylase}

The enzymes transglycosidases, synthases (sugar nucleic acid ester transferases) and phosphorylases can be used 
for carbohydrate synthesis. Transglycosidases catalyze the transfer of glycoside groups from one glycan to another; however, although they do not require the use of expensive substrates, such as nucleotides and glycosyl 1-phosphate, in general they are not suitable for synthesizing high-molecular weight polysaccharides. Synthases catalyze the irreversible addition of glucosyl chains in the glycogen synthesis in vivo; however, the substrate sugar nucleotides are very expensive and impractical. In contrast, phosphorylases catalyze the phosphorolytic reaction and can enable the synthesis of glycosyl chains by reverse reaction from the substrate glycosyl 1-phosphate. Glycosyl 1-phosphate is expensive, but a method has been developed for continuously synthesizing the target glycosyl chain from an inexpensive raw material by combining two types of phosphorylase [1]. Recent advanced research on phosphorylases has identified some with various substrate specificities, and studies on carbohydrate synthesis with these phosphorylases are ongoing [2-4].

\subsection{Synthesis of $\alpha-1,4$ glucose polymers with phosphorylase}

The enzyme glucan phosphorylase catalyzes the $\alpha-1,4$ phosphorolysis of glycosides, as well as the reverse reaction, and can be used to synthesize $\alpha$-1,4-glucan by using glucose 1-phosphate (Glc1P) as a substrate [5-7]. However, this method is impractical because it needs a large amount of expensive Glc1P to extend the glucan chain having high-molecular weight. We are pleased to report that we have developed an effective method to solve this problem. First, we improved the thermal stability of sucrose phosphorylase and glucan phosphorylase $[8,9]$. Next, as shown in Figure 1A, we used sucrose phosphorylase to act on sucrose and produce Glc1P by a phosphorylation reaction; Glc1P then acted as a substrate for the glucan synthesis reaction catalyzed by glucan phosphorylase. The phosphate released in the glucan synthesis reaction is reused in the phosphorolytic reaction catalyzed by sucrose phosphorylase. By combining the two phosphorylases, amylose synthesis can proceed at low phosphate concentrations. In addition, the synthesized glucan chain can be used as a receptor substrate for further elongation, so the molecular weight can be strictly controlled by adjusting the molar ratio of sucrose and the acceptor. In this way, we are able to mass produce amylose with a very narrow molecular weight dispersion $[10,11]$.
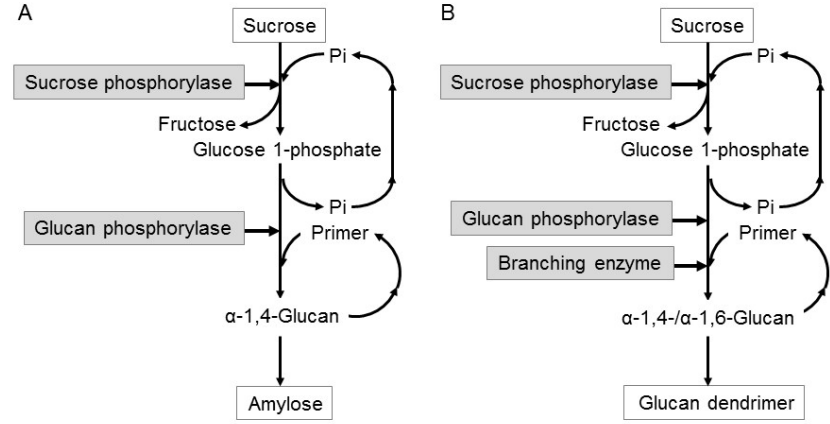

Figure 1: Synthesis of glycogen-like polysaccharides combining phosphorylase and enzymes belonging to the $\alpha$-amylase family. (A) Synthesis of amylose. (B) Synthesis of glucan dendrimer, a glycogen-like polysaccharide that can be synthesized by this method.

\subsection{Synthesis of glycogen-like polysaccharides by combining phosphorylase and branching enzyme}

We achieved the enzyme production of a glycogen-like polysaccharide by using sucrose as a substrate and adding a branching enzyme to the phosphorylase reaction system used for amylose synthesis [12,13] (Fig. 1B). Enzymatically synthesized glycogen-like polysaccharides can be used as materials for foods and cosmetics. They have been reported to have some resistance to digestive enzymes, to be clearly decomposed less than starch, and also to have an immunostimulatory effect when administered orally in animal models $[14,15]$. Similar to the enzymatically synthesized amylose reaction system shown in Figure 1A, our reaction system with phosphorylase and branching enzyme allows the molecular weight of the synthesized glycogen-like polysaccharides to be controlled by the molar ratio of sucrose to primer. We aimed to develop new applications, e.g., for the pharmaceutical field, by increasing the purity of glycogen-like polysaccharides. We were able to produce four glycogen-like polysaccharides with a molecular weight about from $120 \mathrm{kDa}$ to 6,000 $\mathrm{kDa}$ and a molecular weight distribution (weight average molecular weight/number average molecular weight) of 1.1 or less. Small-angle X-ray scattering in an aqueous solution of these glycogen-like polysaccharides showed that all four have structures close to spheres. The inertial radius size of each scattering curve was shown to increase in proportion to the molecular weight, and investigations confirmed from the profile of each curve that the sample was monodisperse [16]. Atomic force microscopy also showed that the glycogen-like polysaccharide particles were monodisperse and spherical [17]. The average 
chain length of the glycogen-like polysaccharides, as determined by the enzymatic method, was about 10 . The glycogen-like polysaccharide synthesized by this method was designated a glucan dendrimer (GD) because it is a spherical, highly branched glucan composed of $\alpha-1,4 / \alpha-1,6$ bonds.

\section{Non-reducing end modification by glucan phosphorylase}

\subsection{Non-reducing end modification of GD}

Chemically synthesized dendrimers, which have numerous linked residues on their surface that are capable of conjugating various functional substances, are being investigated for use as drug delivery system (DDS) carriers, clinical diagnostic markers, and chemical sensors. However, the higher the molecular weight of the dendrimer, the more difficult the chemical synthesis is and the more expensive the materials are. Furthermore, mass production and safety are considered to be problematic. On the other hand, GD is composed of glucose, is a safe, biodegradable material and can be mass produced by the method described above. However, the structure of the dendrimer cannot be effectively utilized with the glucose polymer as it is. Therefore, we attempted to functionalize GD by using functional sugar chains to selectively modify numerous non-reducing ends on the surface of GD particles. The original substrate of glucan phosphorylase is Glc1P (Fig. 2A), but glucan phosphorylase from potato (PotGP) also acts on mannose 1-phosphate (Man1P) and glucosamine 1-phosphate (GlcN1P). Studies have found that mannose and glucosamine can be incorporated into oligosaccharides and that one such monosaccharide residue is attached at the non-reducing end [18-20]. The reaction catalyzed by phosphorylase is an excellent method for non-reducing end modification of GD. Therefore, we investigated the action of glucan phosphorylase on various Glc1P analogs in detail by using maltooligosaccharides as primers [21-23].

\subsection{Action on glucuronic 1-phosphate}

When glucan phosphorylases of various origins acted on chemically synthesized glucuronic acid 1-phosphate (GlcA1P), glucan phosphorylase from Aquifex aeolicus (AqGP) was found to cause a glucuronic acid transfer reaction [24] (Fig. 2B). By analyzing the reaction product by matrix-assisted laser desorption ionization time-offlight mass spectrometry (MALDI-TOF MS) and ${ }^{1} \mathrm{H}$ NMR spectroscopy, we showed that one GlcA residue was linked via an $\alpha-1,4$-linkage to the non-reducing end of the maltooligosaccharide used as a primer. In addition, after digesting the reaction product with glucoamylase (GA), which cleaves maltooligosaccharides into glucose in a stepwise manner from the non-reducing end, highperformance anion-exchange chromatography with pulsed amperometric detection (HPAEC-PAD) analysis revealed that all maltooligosaccharides were decomposed into glucose before the reaction but that the GlcA conjugate was not degraded at all. These results showed that AqGP can use GlcA1P as a donor to bind one molecule of GlcA to the non-reducing end of maltooligosaccharide. By contrast, a similar reaction was not observed when PotGP was used, indicating the high flexibility of donor recognition by AqGP.

\subsection{Action on glucosamine 1-phosphate and mannose 1-phosphate}

As mentioned above, PotGP was reported to act on GlcN1P and Man1P to synthesize oligosaccharides, in which one molecule of mannose or glucosamine was linked to the non-reducing end. However, the receptor recognition of PotGP was strict, and PotGP was not able to recognize maltooligosaccharides, in which either one molecule of mannose or one molecule of glucosamine was linked to the non-reducing end as a receptor. In contrast, when AqGP acted on Man1P as a donor and maltooligosaccharide as an acceptor, oligosaccharides were synthesized, in which multiple mannose residues were linked to the nonreducing ends [25]. A similar reaction occurred when GlcN1P was used as a donor, which established this approach as a novel enzymatic method for synthesizing cationic oligosaccharides with multiple glucosamine residues [26]. These results confirmed that AqGP also shows flexibility in receptor recognition $[22,23]$.

\subsection{Action on galactose 1-phosphate and $\mathrm{N}$-acetylglucosamine 1-phosphate}

We investigated the reactions of AqGP with the other available hexose 1-phosphates, i.e., galactose 1-phosphate (Gal1P) and $N$-acetylglucosamine 1-phosphate (GlcNAc1P). Figure 3 shows the results of HPAEC-PAD analysis of the reaction product when AqGP acts on either $10 \mathrm{mM}$ Gal1P (Fig. 3Aa) or GlcNAc1P (Fig. 3Ba) 
A
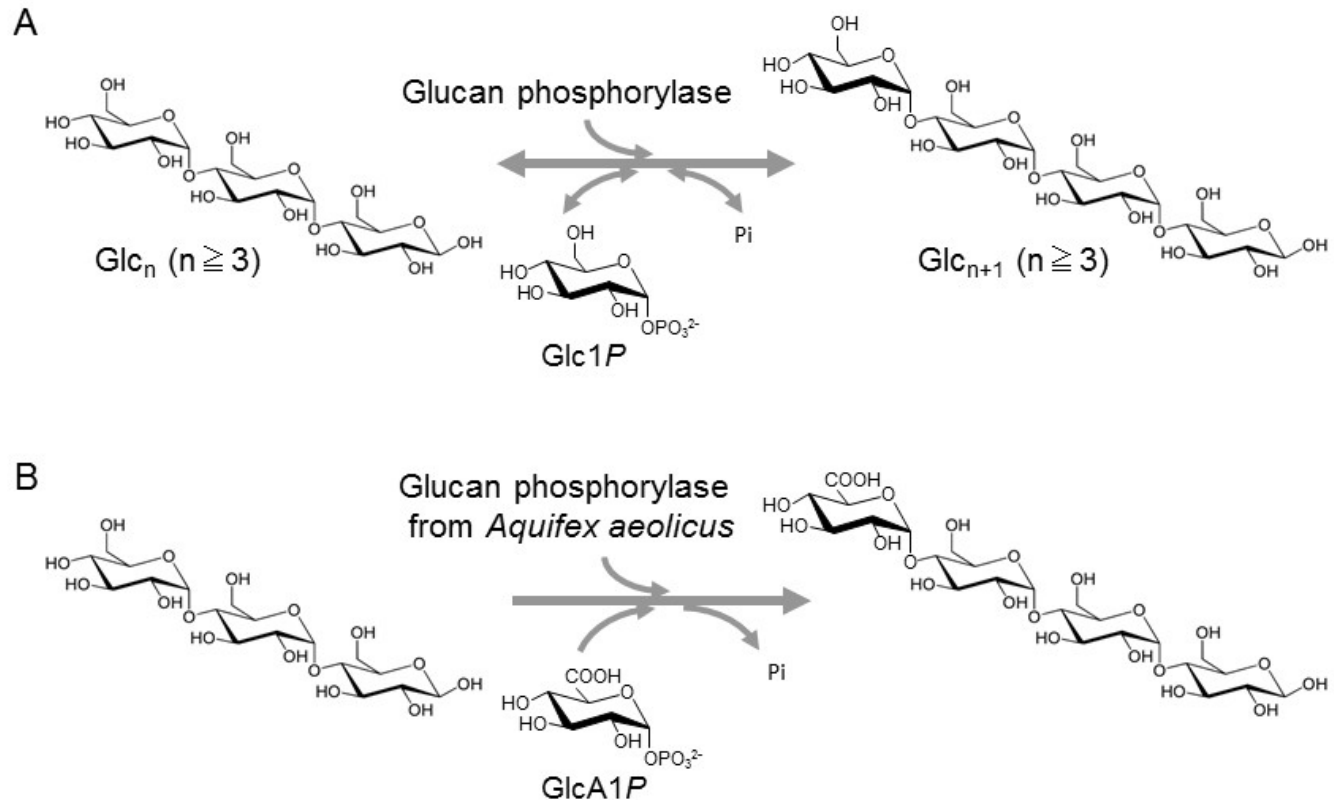

Figure 2: Action of glucan phosphorylase on the substrate glucose 1-phosphate (A) and action of glucan phosphorylase from Aquifex aeolicus on the substrate glucuronic acid 1-phosphate (B). Glc , maltotriose; Glc1P, glucose 1-phosphate; GlcA1P, glucuronic acid 1-phosphate; Pi, inorganic phosphate.

and $5 \mathrm{mM}$ maltotriose $\left(\mathrm{Glc}_{3}\right)$ for $22 \mathrm{~h}$. When Gal1P was used as a donor, the acceptor $\mathrm{Glc}_{3}$ was mostly used up by the end of reaction after the 22-h reaction, and a peak of Gal-Glc ${ }_{3}$ was detected, in which one galactose (Gal) residue was linked to $\mathrm{Glc}_{3}$. AqGP was found to recognize Gal1P as a donor and to link to the non-reducing end of $\mathrm{Glc}_{3}$ (Fig. 3Ab). Interestingly, at the same time, peaks of maltooligosaccharides larger than those of maltose $\left(\mathrm{Glc}_{2}\right)$ were detected. Presumably, this reaction generates $\mathrm{Glc}_{2}$ and Glc1P by a phosphorolytic reaction of $\mathrm{Glc}_{3}$, and the reaction between Glc1P and $\mathrm{Glc}_{3}$ synthesizes maltooligosaccharides larger than maltotetraose $\left(\mathrm{Glc}_{4}\right)$. When the reaction mixture was digested with GA, multiple peaks with Gal residues that were not degraded by GA were detected (Fig. 3Ac). After subsequent purification of this Gal-modified oligosaccharide with an activated carbon column (Fig. 3Ad) and digestion with bacterial $\alpha$-amylase, glucose (Glc), $\mathrm{Glc}_{2}$, and one type of Gal-linked oligosaccharide peak were detected (Fig. 3Ae). These results suggest that AqGP links one Gal residue to the non-reducing end of maltooligosaccharide by using Gal1P as a donor and that, at the same time, a phosphorolytic reaction and a synthetic reaction of $\mathrm{Glc}_{3}$ occur. The same results were found for GlcNAc1P (Fig. 3B). So far, the smallest substrate for the phosphorylation reaction of AqGP was reported to be $\mathrm{Glc}_{4}$, and the smallest receptor for the synthetic reaction was $\mathrm{Glc}_{3}$ [27]. When we used an analog substrate of Glc1P, in particular a hexose that links only one molecule to the non-reducing end, we detected a reaction that could not be detected when using Glc1P as a substrate. Next, we investigated whether a smaller receptor, $\mathrm{Glc}_{2}$, could be recognized by AqGP: AqGP was allowed to act on $5 \mathrm{mM} \mathrm{Glc}_{2}$ or $\mathrm{Glc}_{3}$ for $48 \mathrm{~h}$, with $10 \mathrm{mM}$ Gal1P or GlcNAc1P as a donor, and the phosphate released from Gal1P or GlcNAc1P was quantified over time (Fig. 4). Although the reactivity of $\mathrm{Glc}_{2}$ was much lower than that of $\mathrm{Glc}_{3}$, the amount of phosphate in the reaction solution increased, suggesting that $\mathrm{Glc}_{2}$ is a receptor for AqGP. HPAEC-PAD analysis was performed on each reaction product (Fig. 5). The products of the reaction mixture with Gal1P are shown in Figure 5Aa, and the products of the reaction mixture with GlcNAc1P in Figure 5Ba. When Gal1P was used as the donor, a peak that appeared to be Gal-Glc ${ }_{2}$ was detected, and AqGP was found to recognize $\mathrm{Glc}_{2}$ as an acceptor and to link the Gal residue (Fig. 5Ab). This reaction mixture was digested with GA to decompose unreacted $\mathrm{Glc}_{2}$ into glucose (Fig. 5Ac), then Gal-Glc was purified on an activated carbon column (Fig. 5Ad). When the purified Gal-Glc ${ }_{2}$ was digested with bacterial $\alpha$-amylase, a Glc- and Gal-linked oligosaccharide (GalGlc) peak was detected (Fig. 5Ae). On the other hand, when GlcNAc1P was used as the donor, a peak that appeared to be GlcNAc-Glc ${ }_{2}$ was also detected, and AqGP was found to recognize $\mathrm{Glc}_{2}$ as an acceptor and to link the 
A

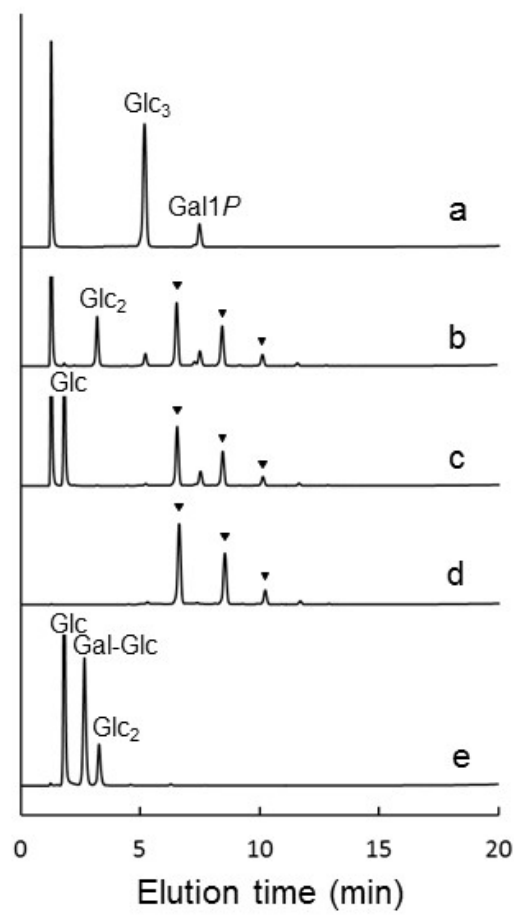

B

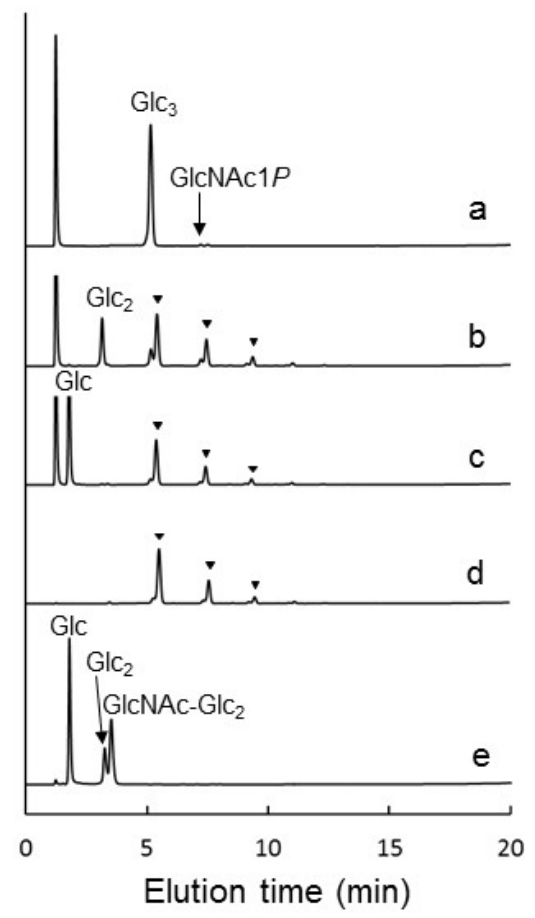

Figure 3: High-performance anion-exchange chromatography with pulsed amperometric detection (HPAEC-PAD) analysis of the products of the action of glucan phosphorylase from Aquifex aeolicus (AqGP) on maltotriose. The reaction mixture (a) including $10 \mathrm{mM}$ galactose 1-phosphate (A) or $\mathrm{N}$-acetylglucosamine 1-phosphate (B) with $5 \mathrm{mM}$ maltotriose and $100 \mathrm{mM}$ acetate buffer (pH 5.5), was treated by AqGP (40 $\mathrm{U} / \mathrm{mL}$ ) at $55^{\circ} \mathrm{C}$ for $22 \mathrm{~h}$. HPAEC-PAD was used to analyze the reaction products before any treatment (b), after glucoamylase (GA) treatment (c), after GA treatment and carbon column treatment (d), and after GA, carbon column, and $\alpha$-amylase treatment (e). Gal1P, galactose 1-phosphate; GlcNAc1P, $\mathrm{N}$-acetylglucosamine 1-phosphate; Glc, glucose; Glc ${ }_{2}$, maltose; $\mathrm{Glc}_{3}$, maltotriose; $\boldsymbol{\nabla}$, products of AqGP treatment.
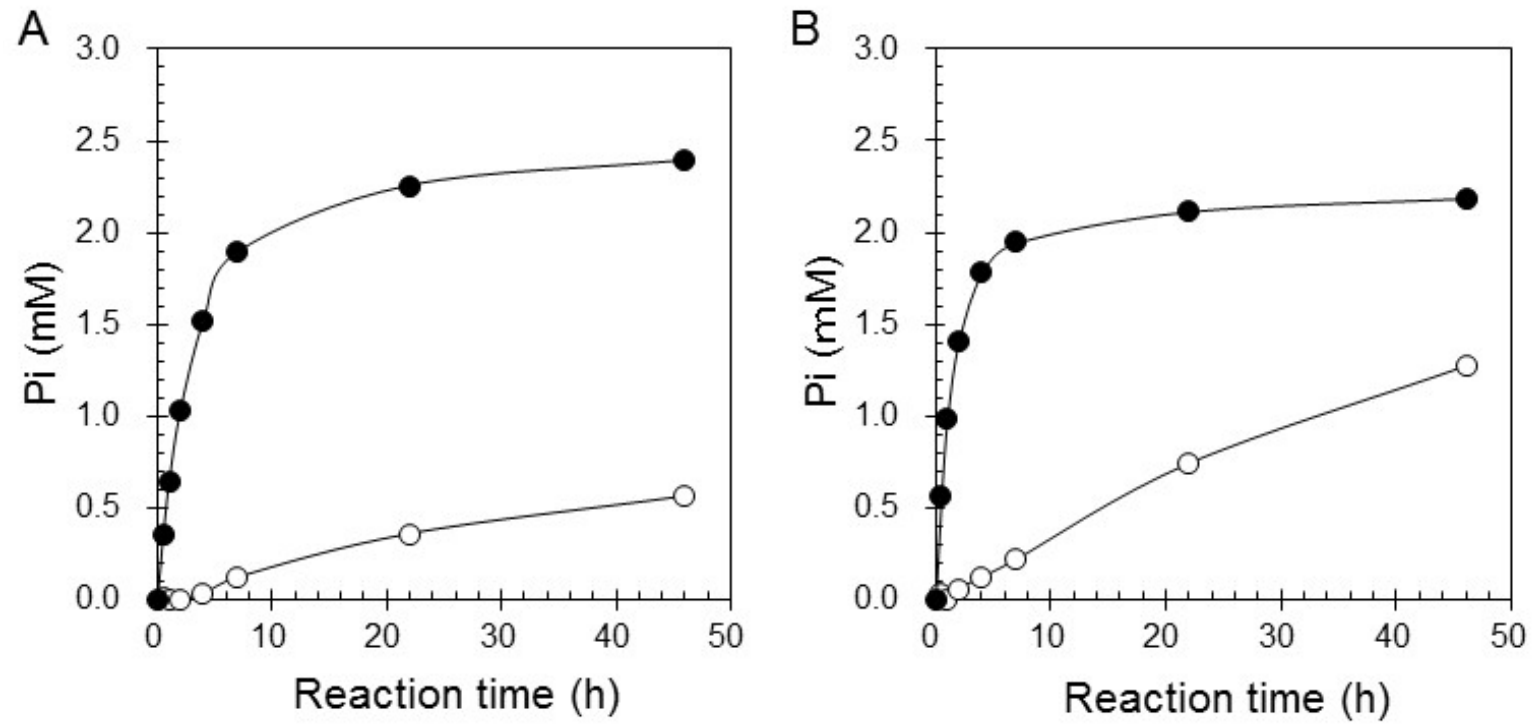

Figure 4: Modification reaction of glucan phosphorylase from Aquifex aeolicus (AqGP) with maltose and maltotriose. The reaction mixture comprising $10 \mathrm{mM}$ galactose 1-phosphate (A) or $\mathrm{N}$-acetylglucosamine 1-phosphate (B) with $5 \mathrm{mM}$ acceptor (maltotriose [0] or maltose [O]) and $100 \mathrm{mM}$ acetate buffer $\left(\mathrm{pH}\right.$ 5.5) was treated by AqGP $(40 \mathrm{U} / \mathrm{mL})$ at $50^{\circ} \mathrm{C}$ and the change in inorganic phosphate levels over time were measured. 

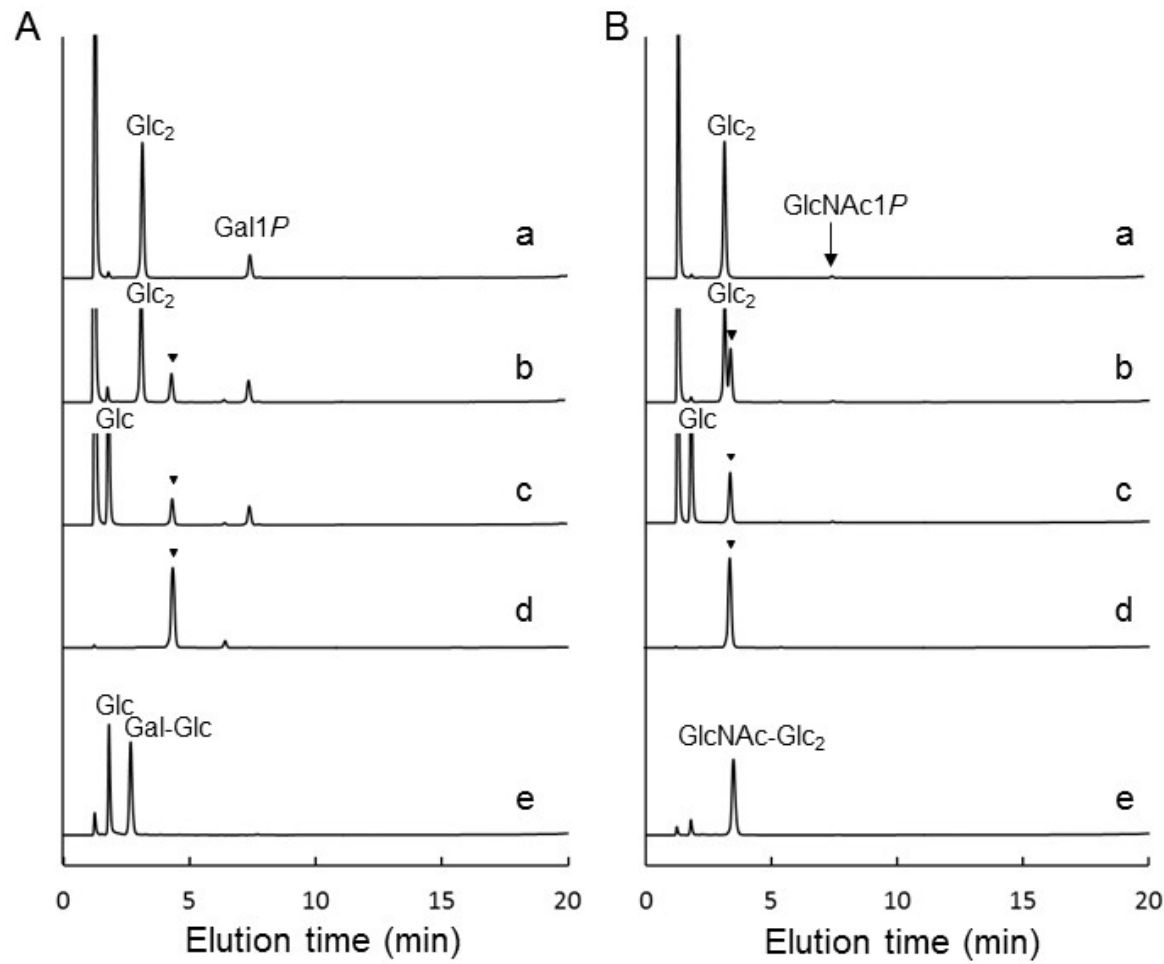

Figure 5: High-performance anion-exchange chromatography with pulsed amperometric detection (HPAEC-PAD) analysis of the products of glucan phosphorylase from Aquifex aeolicus (AqGP) on maltose. The reaction mixture (a) including $10 \mathrm{mM}$ galactose 1-phosphate (A) or $\mathrm{N}$-acetylglucosamine 1-phosphate (B) with $5 \mathrm{mM}$ maltose and $100 \mathrm{mM}$ acetate buffer $(\mathrm{pH} 5.5)$ were treated by $\mathrm{AqGP}(40 \mathrm{U} / \mathrm{mL})$ at $50{ }^{\circ} \mathrm{C}$ for 48 h. HPAEC-PAD was used to analyze the products of AqGP before any treatment (b), after glucoamylase (GA) treatment (c), after GA and carbon column treatment (d), and after GA, carbon column and $\alpha$-amylase treatment (e). Gal1P, galactose 1-phosphate; Glc, glucose; Glc ${ }_{2}$, maltose; Gal-Glc, galactose-glucose; GIcNAc1P, $N$-acetylglucosamine 1-phosphate; GlcNAc-Glc,$N$-acetylglucosamine-maltose; $\nabla$, products of AqGP treatment.

$N$-acetylglucosamine (GlcNAc) residue (Fig. 5Bb). This reaction mixture was digested with GA to decompose unreacted $\mathrm{Glc}_{2}$ into glucose (Fig. 5Bc), then GlcNAc$\mathrm{Glc}_{2}$ was purified on an activated charcoal column (Fig. 5Bd). When the purified GlcNAc-Glc ${ }_{2}$ was digested with bacterial $\alpha$-amylase, cleavage by bacterial $\alpha$-amylase did not occur, and a peak that was presumed to be GlcNAc$\mathrm{Glc}_{2}$ was detected (Fig. 5Be). The purified GlcNAc-Glc ${ }_{2}$ was analyzed by MALDI-TOF MS and ${ }^{1} \mathrm{H}$ NMR spectroscopy, which confirmed that the assessed structure matched the estimated structure. These results show that AqGP recognizes five types of hexose 1-phosphate, i.e., GlcA1P, GlcN1P, Man1P, Gal1P, and GlcNAc1 $P$, as donors and adds glucuronic acid, glucosamine, mannose, galactose, and $\mathrm{N}$-acetylglucosamine, respectively, on the non-reducing ends of maltooligosaccharides. They also show that AqGP is flexible in not only donor but also receptor recognition, with $\mathrm{Glc}_{3}$ being the smallest substrate for phosphorylation degradation and Glc ${ }_{2}$ being the smallest receptor.

\subsection{Modification of the non-reducing ends of GD}

We investigated whether AqGP can react with GD, a highmolecular weight branched polysaccharide, to transfer hexose rather than maltooligosaccharide to the nonreducing end of GD [28]. We examined the amount of phosphate liberated by the action of AqGP on $20 \mathrm{mM}$ of seven different hexose 1-phosphates and $20 \mathrm{mM} \mathrm{GD}$, and found that GlcA1P, GlcN1P, Man1P, Gal1P, and GlcNAc1P acted as substrates (Fig. 6), although they were less reactive than Glc1P. In contrast, AqGP hardly reacted with galactosamine 1-phosphate (GalN1P). The fact that these hexoses were linked to the non-reducing ends was confirmed by HPAEC-PAD analysis of the chain-length distribution after debranching of GD. GD, with a glucose molecule on the non-reducing end, was completely decomposed into glucose when treated with exo-type amylase after debranching (Fig. 7A), whereas GD with a hexose other than glucose on the non-reducing end 


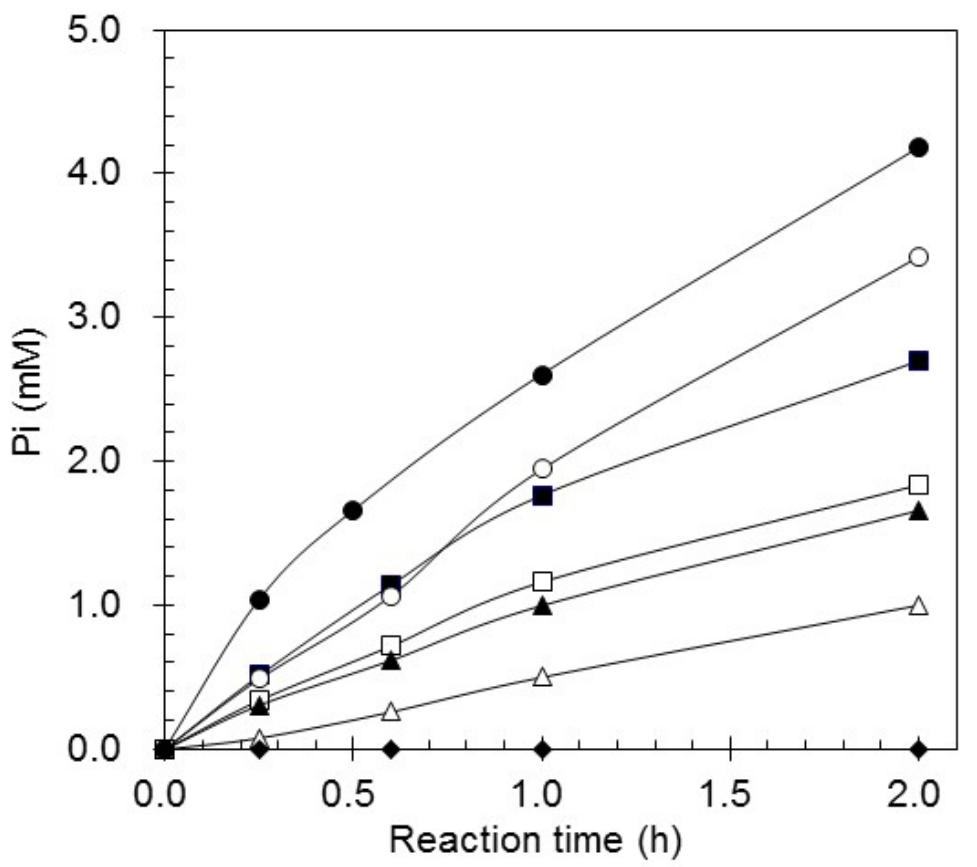

Figure 6: Modification of the non-reducing ends of glucan dendrimer (GD) by using seven different hexose 1-phosphates. The reaction mixture comprising $20 \mathrm{mM}$ hexose 1-phosphate and 20 mM GD10 (molecular weight, 121,800) and $100 \mathrm{mM}$ acetate buffer (pH 5.5), was treated by glucan phosphorylase from Aquifex aeolicus $\left(40 \mathrm{U} / \mathrm{mL}\right.$ or $0.05 \mathrm{U} / \mathrm{mL}$ ) at $55^{\circ} \mathrm{C}$ for 0.25 to $2 \mathrm{~h}$ and the inorganic phosphate produced was measured. The following hexose 1-phosphates were used: glucose-1-phosphate ( $(\mathbf{)})$, glucosamine 1-phosphate (O), glucuronic acid 1-phosphate $(\square), N$-acetylglucosamine 1-phosphate $(\square)$, mannose 1-phosphate $(\boldsymbol{\Delta})$, galactose 1-phosphate $(\triangle)$, and galactosamine 1-phosphate $(\diamond)$. $\mathrm{Pi}$, inorganic phosphate.

was not degraded by exo-type amylase and showed a characteristic pattern (Figs. 7B to F). These findings indicate that glucuronic acid, glucosamine, mannose, galactose, and $N$-acetylglucosamine can be added to the non-reducing ends of GD by $\alpha-1,4$ glycosidic linkages, as in the case of maltooligosaccharides. Next, we investigated whether the modification of the non-reducing end GD could be controlled and whether the particle size of GD affected the control of this modification. To do so, four types of GD with different particle sizes (GD10, GD20, GD30, and GD40) were modified with hexose 1-phosphate, and their modification ratios were compared. The results with GlcA1P are shown in Figure 8. By adjusting the molar ratio of GlcA1P to the non-reducing end of GD, glucuronic acid was able to link to, i.e., modify, approximately $70 \%$ of the non-reducing ends of GD10. For larger molecular sizes, such as GD30 and GD40, modification was limited to about $30 \%$. Thus, the proportion of non-reducing ends present on the particle surface, on which AqGP can act, decreases as the molecular size increases, but about 30\% of the non-reducing terminals can be modified without affecting the size of GD.

\section{Functionalization of GD by modification of glucuronic acid and glucosamine}

When AqGP acted on GlcA1P or GlcN1P as a substrate, the non-reducing ends of GD40 were modified by about $10 \%$ with glucuronic acid and by $20 \%$ with glucosamine. When the zeta potential of the unmodified and modified GD40 particles was measured, unmodified GD40 had a potential of almost 0 , whereas GD40 modified by glucuronic acid showed a negative charge and GD40 modified by glucosamine exhibited a positive one. The charge increased as the terminal modification rate increased and showed a tendency to become stronger (Fig. 9). Such GD particles with negative and positive charges on the surface are thought to interact electrostatically with substances that have a positive or negative charge, respectively [2931]. Therefore, we expect that nucleic acids and proteins could be bound to the surface of GD particles via charge interactions, and we were able to confirm this hypothesis (data not shown). In addition, glucuronic acid modification and glucosamine modification mean that carboxyl groups and amino groups are introduced into the non-reducing 

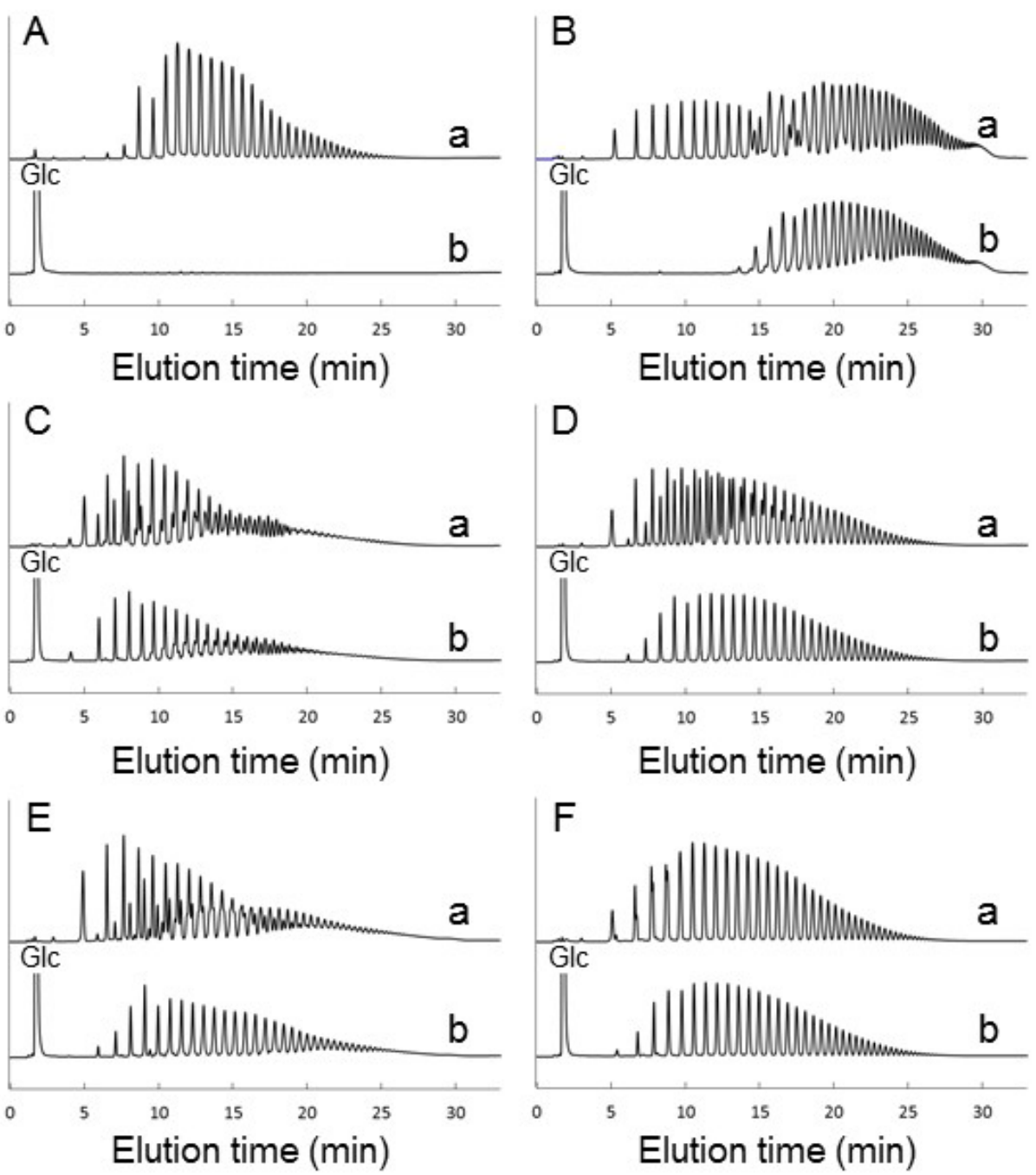

Figure 7: High-performance anion-exchange chromatography with pulsed amperometric detection (HPAEC-PAD) analysis of glucan dendrimer (GD) modified with hexose 1-phosphate by using glucan phosphorylase from Aquifex aeolicus. The modified GDs were incubated at $50{ }^{\circ} \mathrm{C}$ for $20 \mathrm{~h}$ in the same conditions as described in the legend of Figure 6. The products were debranched with isoamylase and pullulanase (a) and treated with $\alpha$-amylase (b). Part (A) shows the results before modification, whereas parts (B) to (F) show the results after the following modifications: (B), glucronic acid 1-phosphate; (C), glucosamine 1-phosphate; (D), mannose 1-phosphate; (E), galactose 1-phosphate; (F), $\mathrm{N}$-acetylglucosamine 1-phosphate. Glc, glucose.

ends of GD, respectively. The introduction of these groups into the non-reducing ends of GD enables linking between the surface of GD particles and other functional molecules via chemical bonding. Specifically, the carboxyl groups introduced at the non-reducing end of GD are covalently conjugated to a functional substance with an amino group, such as a protein or peptide, by activating the substance with $N$-hydroxysuccinimide ester. Furthermore, the amino groups introduced at the GD non-reducing end can be used as they are or in combination with various linkers for covalent conjugating of various functional substances. The non-reducing end-modification technology of GD that we have developed can introduce carboxyl groups and amino groups at the desired percentage of non-reducing ends, which is not possible in currently marketed dendrimers, like polyamidoamine that are synthesized chemically. Thus, the applicability of functionalized GD to pharmaceutical applications can be enhanced by combining non-reducing end-modification and chemical synthesis technologies. 


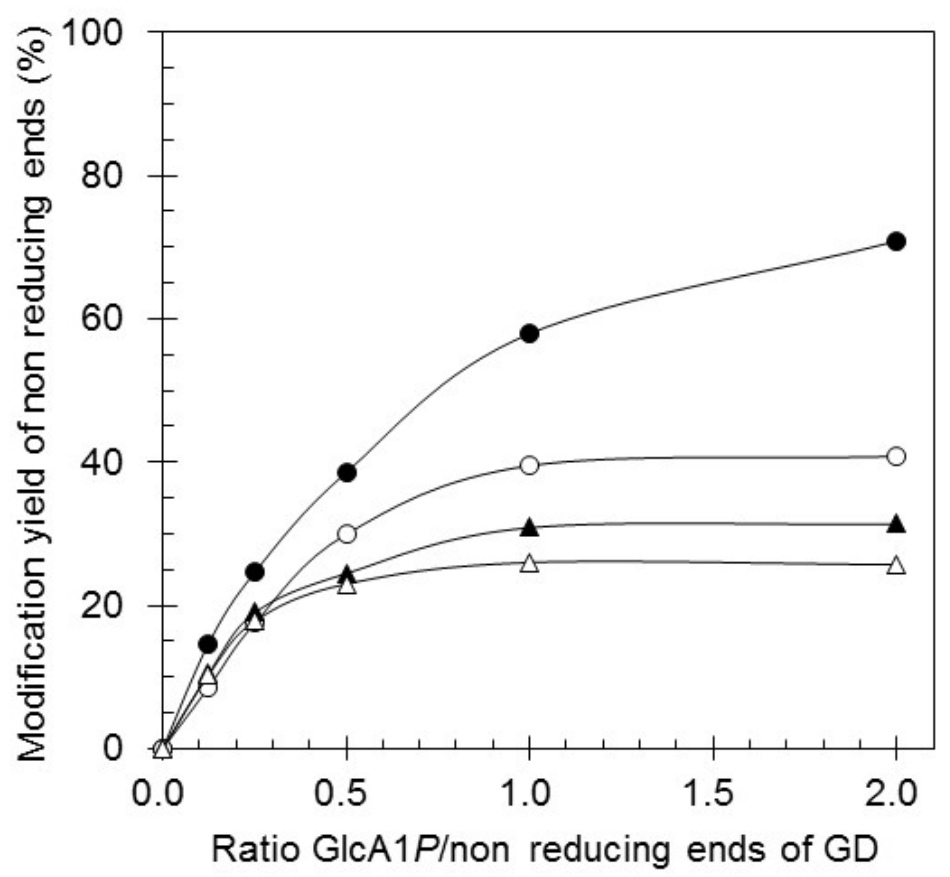

Figure 8: Modification of the surface of different sizes of glucan dendrimer (GD). The reaction mixture comprising $2 \mathrm{mM}$ GDs with 0.25 to $4 \mathrm{mM}$ and $100 \mathrm{mM}$ acetate buffer $\left(\mathrm{pH}\right.$ 5.5) was treated by glucan phosphorylase from Aquifex aeolicus $(40 \mathrm{U} / \mathrm{mL})$ at $50^{\circ} \mathrm{C}$ for $24 \mathrm{~h}$, and the modification ratio of glucuronic acid was measured. The molecular weight of each GD was analyzed by size exclusion chromatography with multi-angle light scattering (SEC-MALS) and particle sizes were measured by dynamic light scattering (DLS). The horizontal axis shows the ratio of glucuronic acid 1-phosphate to the non-reducing end of the GD, and the vertical axis shows the glycosylation ratio. The GDs used

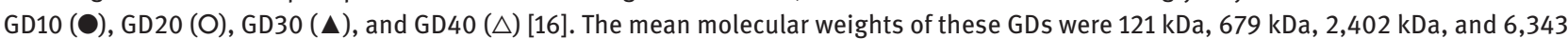
$\mathrm{kDa}$, respectively, and the corresponding particle sizes were $15.8 \mathrm{~nm}, 20.1 \mathrm{~nm}, 28.3 \mathrm{~nm}$ and $37.3 \mathrm{~nm}$. GlcA1P, glucuronic acid 1-phosphate; $\mathrm{GD}$, glucan dendrimer.

\section{Application of GD to pharmaceutical applications}

Recent developments in cancer immunotherapy require administered antigen peptides and other drugs to be transported to lymph nodes and taken up by localized antigen-presenting cells [32,33]. Nano-sized spherical particles were found to be suitable for targeting to lymph nodes [33,34], and in an animal experiment [34], high-molecular weight micelles with a particle size of about $30 \mathrm{~nm}$ that contained an anticancer drug were intravenously administered to target lymph nodes. Studies $[33,34]$ found that the growth of cancer that has spread to the lymph nodes can be effectively suppressed by GD particles synthesized with a controlled particle size, and researchers generated GD30 with a particle size of about $30 \mathrm{~nm}$. Non-reducing end-selective modification of GD30 allows drugs, such as anticancer agents to be linked to the surface of GD30 particles. GD with a particle size adjusted in this way is expected to be suitable for use as a DDS carrier, such as for lymph node delivery.
In addition, nanoparticles, in which the surface layer of polymer micelles is cationized or anionized, were found to be effectively taken up by antigen-presenting cells [3537], so further applications of antigen presentation by GD particles, in which the non-reducing ends are modified with glucuronic acid or glucosamine, can be expected. On the other hand, non-reducing end-modified GD particles can be used as excellent carriers for various sugar chains at

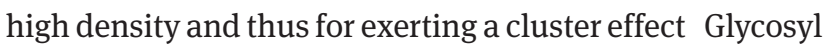
chains play various physiological functions, such as cell adhesion and signal transduction. However, the binding between glycosyl chains and their acceptors is weak, and increasing the density of glycosyl chains was reported to be important to exert a cluster effect and thus fully make use of the functions of glycosyl chains [38-44]. GD particles have a large number of non-reducing ends, and a large number of glycosyl chains can be accumulated at high density on the surface of GD particles by using nonreducing end-modification technology. Thus, GD particles are excellent carriers for exerting the cluster effect of glycosyl chains. 


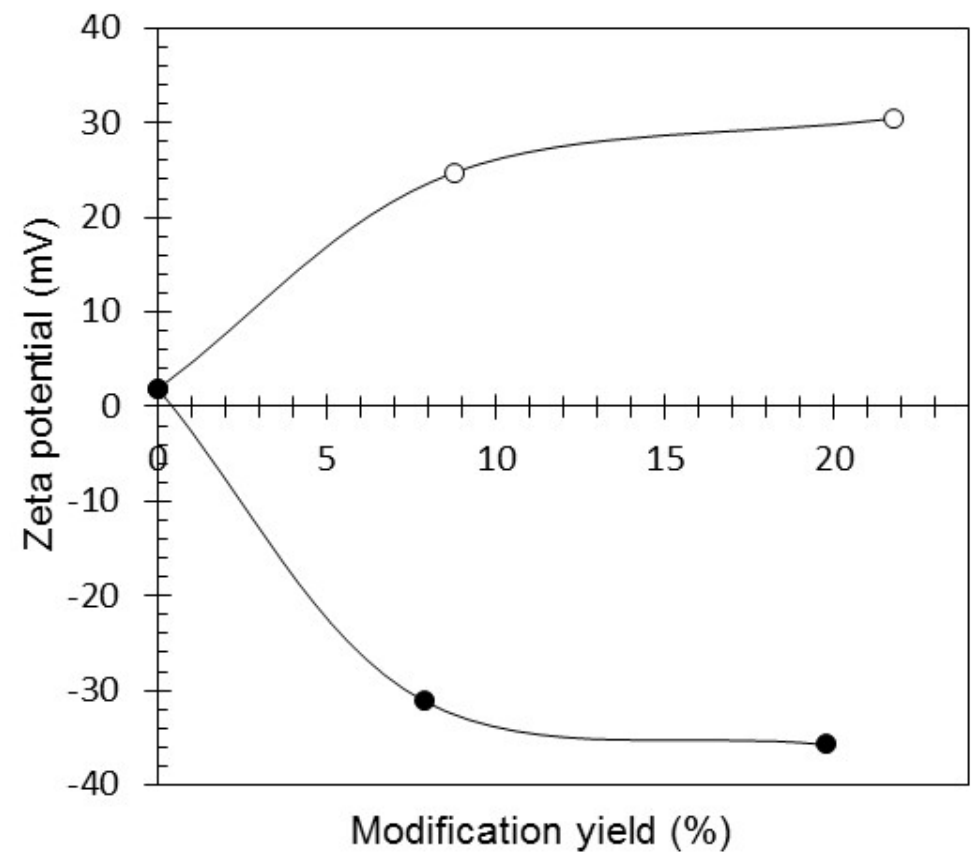

Figure 9: The surface charge of glucan dendrimers (GDs) modified with glucuronic acid and glucosamine. The zeta potential of the surface charge was measured with ELSZ-1 (Otsuka Electronics Co., Ltd.). The GD40 used in the reaction shown in Figure 8 was modified with glucuronic acid (GlcA) or glucosamine (GlcN). Each $1 \mathrm{mg} / \mathrm{mL}$ of modified GD40 was dissolved in $0.01 \mathrm{mM}$ sodium phosphate buffer ( $\mathrm{pH} 7.0$ ) and measured. The horizontal axis shows the modification rate of GIcA or GIcN, and the vertical axis shows the zeta potential. $\bullet$, GD40 modified with GICA; O, GD40 modified with GICN.

\section{Conclusion}

The medicinal properties of pharmaceuticals have changed significantly from synthetic, low-molecular weight compounds to biological constituents, such as proteins, peptides, and antibodies, and their properties are expanding to the use of nucleic acids and cells. So far, pharmaceutical companies have shown little interest in using polysaccharides as a component of pharmaceutical products; however, this may change with the advent of GD and its non-reducing end-modification technology, which are excellent platforms for the development of DDS carriers and polysaccharide chain drugs. Currently, we are promoting the possibility of using GD for such purposes.

Conflict of interest: The authors declare no conflict of interest.

\section{References}

[1] Kitaoka M., pp. 401-404. In: Handbook of Advanced Glycoscience and Glycoengineering, NTS, Tokyo, 2015.

[2] Kitaoka M., Tian J., Nishimoto M., A novel putative galactose operon involving lacto- $\mathrm{N}$-biose phosphorylase found in
Bifidobacterium longum. Appl. Environ. Microbiol. 2005, 71, 3158-3162. https://doi.org/10.1128/AEM.71.6.3158-3162.2005

[3] Senoura T., Ito S., Taguchi H., Higa M., Hamada S., Matsui H., Ozawa T., Jin S., Watanabe J., Wasaki J., Ito S., New microbial mannan catabolic pathway that involves a novel mannosylglucose phosphorylase. Biochem. Biophys. Res. Commun. 2011, 408, 701-706. https://doi.org/10.1016/j. bbrc.2011.04.095

[4] Nihira T., Suzuki E., Kitaoka M., Nishimoto M., Ohtsubo K., Nakai H., Discovery of $\alpha-1,4-d$-mannosyl- $N$-acetyl-dglucosamine phosphorylase involved in the metabolism of N-glycans. J. Biol. Chem. 2013, 288, 27366-27374. https://doi. org $/ 10.1074 /$ jbc.M113.469080

[5] Kitamura S., Starch polymers, natural and synthetic, pp. 7915-7922. In: Salamone J.C. (Ed.) The Polymeric Materials Encyclopedia Synthesis: Properties and Applycation, CRC Press, Boca Raton. 1996.

[6] Fujii K., Takata H., Yanase M., Terada Y., Ohdan K., Takaha T., Okada S., Kuriki T., Bioengineering and application of novel glucose polymers. Biocatal. Biotransform. 2003, 21, 167-172. https://doi.org/10.1080/10242420310001614379

[7] Waldmann H., Gygax D., Bednarski M.D., Shangraw W.R., Whitesides G.M., The enzymic utilization of sucrose in the synthesis of amylose and derivatives of amylose, using phosphorylases. Carbohydr. Res. 1986, 157, c4-c7. https://doi. org/10.1016/0008-6215(86)85078-9

[8] Fujii K., liboshi M., Yanase M., Takaha T., Kuriki T., Enhancing the thermal stability of sucrose phosphorylase from 
Streptococcus mutans by random mutagenesis. J. Appl. Glycosci. 2006, 53, 91-97. https://doi.org/10.5458/jag.53.91

[9] Yanase M., Takata H., Fujii K., Takaha T., Kuriki T., Cumulative effect of amino acid replacements results in enhanced thermostability of potato type $L \alpha$-glucan phosphorylase. Appl. Environ. Microbiol. 2005, 71, 5433-5439. https://dx.doi. org/10.1128/AEM.71.9.5433-5439.2005

[10] Ohdan K., Fujii K., Yanase M., Takaha T., Kuriki T., Enzymatic synthesis of amylose. Biocatal. Biotransform. 2006, 24, 77-81. https://doi.org/10.1080/10242420600598152

[11] Yanase M., Takaha T., Kuriki T., $\alpha$-Glucan phosphorylase and its use in carbohydrate engineering. J. Sci. Food Agric. 2006, 86, 1631-1635. https://doi.org/10.1002/jsfa.2513

[12] Kajiura H., Kakutani R., Akiyama T., Takata H., Kuriki T., A novel enzymatic process for glycogen production. Biocatal. Biotransform. 2008, 26, 133-140. https://doi. org/10.1080/10242420701789411

[13] Takata, H., Ohdan, K., Takaha, T., Kuriki, T., Okada, S., Properties of branching enzyme from hyperthermophilic bacterium, Aquifex aeolicus, and its potential for production of highly-branched cyclic dextrin. J. Appl. Glycosci. 2003, 50, 15-20. https://doi.org/10.5458/jag.50.15

[14] Takata H., Kajiura H., Furuyashiki T., Kakutani R., Kuriki T., Fine structural properties of natural and synthetic glycogens. Carbohydr. Res. 2009, 344, 654-659. https://doi. org/10.1016/j.carres.2009.01.008

[15] Kakutani R., Adachi Y., Kajiura H., Takata H., Kuriki T., Ohno N., The effect of orally administered glycogen on antitumor activity and natural killer cell activity in mice. Int Immunopharmacol. 2012, 12, 80-87. https://doi.org/10.1016/j. intimp.2011.10.017

[16] Kageyama A., Yanase M., Uguchi Y., Structural characterization of enzymatically synthesized glucan dendrimers. Carbohydr. Polym. 2019, 204, 104-110. https://doi.org/10.1016/j. carbpol.2018.09.053

[17] Takeda S., Nishimura T., Umezaki K., Kubo A., Yanase M., Sawada S., Sasaki Y., Akiyoshi K., Synthesis and function of amphiphilic glucan dendrimers as nanocarriers for protein delivery. Biomater. Sci. 2019, 7, 1617-1622. https://doi. org/10.1039/c8bm01627e

[18] Evers B., Thiem J., Further syntheses employing phosphorylase. Bioorg. Med. Chem. 1997, 5, 857-863. https://doi.org/10.1016/ S0968-0896\%2897\%2900031-X

[19] Nawaji M., Izawa H., Kaneko Y., Kadokawa J., Enzymatic $\alpha$-glucosaminylation of maltooligosaccharides catalyzed by phosphorylase. Carbohydr. Res. 2008, 343, 2692-2696. https://doi.org/10.1016/j.carres.2008.08.013

[20] Baba R., Yamamoto K., Kadokawa J., Synthesis of $\alpha(1,4)$-linked non-natural mannoglucans by $\alpha$-glucan phosphorylasecatalyzed enzymatic copolymerization. Carbohydr. Polym. 2016, 151, 1034-1039. https://doi.org/10.1016/j. carbpol.2016.06.057

[21] Kawazoe S., Izawa H., Nawaji M., Kaneko Y., Kadokawa J., Phosphorylase-catalyzed $\mathrm{N}$-formyl- $\alpha$-glucosaminylation of maltooligosaccharides. Carbohydr. Res. 2010, 345, 631-636. https://doi.org/10.1016/j.carres.2010.01.001

[22] Kadokawa J., Glucan phosphorylase-catalyzed enzymatic glycosylations using analogue substrates of $\alpha$-d-glucose 1-phosphate. J. Appl. Glycosci. 2014, 4, 160-166. https://doi. org/10.5458/bag.4.2_160
[23] Kadokawa J., $\alpha$-Glucan phosphorylase: a useful catalyst for precision enzymatic synthesis of oligo- and polysaccharides. Curr. Org. Chem. 2017, 21, 1192-1204. http://dx.doi.org/10.2174 /1385272821666170222124526

[24] Umegatani Y., Izawa H., Nawaji M., Yamamoto K., Kubo A., Yanase M., Takaha T., Kadokawa J., Enzymatic $\alpha$-glucuronylation of maltooligosaccharides using $\alpha$-glucuronic acid 1-phosphate as glycosyl donor catalyzed by a thermostable phosphorylase from Aquifex aeolicus VF5. Carbohydr. Res. 2012, 350, 81-85. https://doi.org/10.1016/j. carres.2011.12.022

[25] Simohigoshi R., Takemoto Y., Yamamoto K. Kadokawa J., Thermostable $\alpha$-glucan phosphorylase-catalyzed successive $\alpha$-mannosylations. Chem. Lett. 2013, 42, 822-824. https://doi. org/10.1246\%2Fcl.130286

[26] Kadokawa J., Shimohigoshi R., Yamashita K. Yamamoto K., Synthesis of chitin and chitosan stereoisomers by thermostable $\alpha$-glucan phosphorylase-catalyzed enzymatic polymerization of $\alpha$-d-glucosamine 1-phosphate. Org. Biomol. Chem. 2015, 13, 4336-4343. https://doi.org/10.1039/ C50B00167F

[27] Bhuiyan S.H., Rus'd A.A., Kitaoka M. Hayashi K., Characterization of a hyperthermostable glycogen phosphorylase from Aquifex aeolicus expressed in Escherichia coli. J. Mol. Catal. B Enzym. 2003, 22, 173-180. https://doi. org/10.1016/S1381-1177\%2803\%2900029-8

[28] Takemoto Y., Izawa H., Umegatani Y., Yamamoto K., Kubo A., Yanase M., Takaha T., Kadokawa J., Synthesis of highly branched anionic $\alpha$-glucans by thermostable phosphorylasecatalyzed $\alpha$-glucuronylation. Carbohydr. Res. 2013, 366, 38-44. https://doi.org/10.1016/j.carres.2012.11.007

[29] Takata Y., Shimohigoshi R., Yamamoto K. Kadokawa J., Enzymatic synthesis of dendritic amphoteric $\alpha$-glucans by thermostable phosphorylase catalysis. Macromol. Biosci. 2014, 14, 1437-1443. https://doi.org/10.1002/mabi.201400204

[30] Yamashita K., Yamamoto K., Kadokawa J., Synthesis of non-natural heteroaminopolysaccharides by $\alpha$-glucan phosphorylase-catalyzed enzymatic copolymerization: $\alpha(1,4)$ linked glucosaminoglucans. Biomacromolecules 2015, 16, 3989-3994. https://doi.org/10.1021/acs.biomac.5b01332

[31] Kadokawa J., Yamashita K., Shimohigoshi R., Yamamoto K., An investigation of enzymatic phosphorolysis of $\alpha$ (1à4)linked oligo-d-glucosaminides by thermostable $\alpha$-glucan phosphorylase catalysis. J. Org. Inorg. Chem. 2016, 2, 3. https://doi.org/10.21767/2472-1123.100010

[32] Kobiyama K., Aoshi T., Narita H., Kuroda E., Hayashi M. Tetsutani K., Koyama S., Mochizuki S., Sakurai K., Katakai Y., Yasutomi Y., Saijo S., Iwakura Y., Akira S., Coban C. Ishii K. J., Nonagonistic dectin-1 ligand transforms $\mathrm{CpG}$ into a multitask nanoparticulate TLR9 agonist. Proc. Natl. Acad. Sci. USA 2014, 111, 3086-3091. https://doi.org/10.1073/pnas.1319268111

[33] Kawakami S., Higuchi Y., Nakanishi H., Kuramoto Y., Hattori Y., Hashida M., Development of cancer immune therapy by cell targeted nucleic acid delivery. Drug Delivery System 2008, 23, 138-144. https://doi.org/10.2745/dds.23.138

[34] Cabral H., Makino J., Matsumoto Y., Mi P., Wu H., Nomoto T., Toh K., Yamada N., Higuchi Y., Konishi S., Kano M.R., Nishihara H., Miura Y., Nishiyama N. Kataoka K., Systemic targeting of lymph node metastasis through the blood vascular system by using 
size-controlled nanocarriers. ACS Nano 2015, 9, 4957-4967. https://doi.org/10.1021/nn5070259

[35] Pittella F., Zhang M., Lee Y., Kim H.J., Tockary T., Osada K., Ishii T., Miyata K., Nishiyama N. Kataoka K., Enhanced endosomal escape of siRNA-incorporating hybrid nanoparticles from calcium phosphate and PEG-block charge-conversional polymer for efficient gene knockdown with negligible cytotoxicity. Biomaterials 2011, 32, 3106-3114. https://doi.org/10.1016/j. biomaterials.2010.12.057

[36] Takemoto H., Ishii A., Miyata K., Nakanishi M., Oba M., Ishii T., Yamasaki Y., Nishiyama N. Kataoka K., Polyion complex stability and gene silencing efficiency with a siRNA-grafted polymer delivery system. Biomaterials 2010, 31, 8097-8105. https:// doi.org/10.1016/j.biomaterials.2010.07.015

[37] Yamamoto S., Yuba E., Harada A. Kono K., Effective condensation of multivalent anions into polyion complex micelles prepared from $\mathrm{TiO}_{2}$ nanoparticles and polyallylamine bearing poly (ethylene glycol) grafts. Langmuir 2015, 31, 8583-8588. https://doi.org/10.1021/acs.langmuir.5b01883

[38] Miura Y., Synthesis and biological application of glycopolymers. J. Polym. Sci. Part A Polym. Chem. 2007, 45, 5031-5036. https://doi.org/10.1002/pola.22369

[39] Hattori Y., Kawakami S., Lu Y., Nakamura Y., Yamashita F., Hashida M., Enhanced DNA vaccine potency by mannosylated lipoplex after intraperitoneal administration. J. Gene Med. 2006, 8, 824-834. https://doi.org/10.1002/jgm.910

[40] Ogata M., Zeng X., Usui T., Uzawa H., Substrate specificity of $\mathrm{N}$-acetylhexosaminidase from Aspergillus oryzae to artificial glycosyl acceptors having various substituents at the reducing ends. Carbohydr. Res. 2007, 342, 23-30. https://doi. org/10.1016/j.carres.2006.11.004

[41] Ogata M., Hidari K.I.P.J., Murata T., Shimada S., Kozaki W., Park E.Y., Suzuki T., Usui T., Chemoenzymatic synthesis of sialoglycopolypeptides as glycomimetics to block infection by avian and human influenza viruses. Bioconjug. Chem. 2009, 20, 538-549. https://doi.org/10.1021/bc800460p

[42] Ogata M., Murata T., Park E.Y., Usui T., Chemoenzymatic synthesis of glycan-arranged polymeric inhibitors against influenza virus infection. J. Appl. Glycosci. 2010, 57, 137-144. https://doi.org/10.5458/jag.57.137

[43] Ogata M., Umemura S., Sugiyama N., Kuwano N., Koizumi A., Sawada T., Yanase M., Takaha T., Kadokawa J., Usui T., Synthesis of multivalent sialyllactosamine-carrying glyconanoparticles with high affinity to the human influenza virus hemagglutinin. Carbohydr. Polym. 2016, 153, 96-104. https:// doi.org/10.1016/j.carbpol.2016.07.083

[44] Chinnathambi S., Chen S., Ganesan S., Hanagata N., Binding mode of $\mathrm{CpG}$ oligodeoxynucleotides to nanoparticles regulates bifurcated cytokine induction via toll-like receptor 9. Sci. Rep. 2012, 2, 534. https://doi.org/10.1038/srep00534 